\title{
Poloidal polarimeter system for current density measurements in ITER
}

\author{
A. J. H. Donné \\ FOM-Instituut voor Plasmafysica Rijnhuizen, Associatie EURATOM-FOM, 3430 BE Nieuwegein, \\ The Netherlands \\ T. Edlington \\ UKAEA Culham Laboratory, Abingdon, Oxon, OX14 3DB, United Kingdom \\ E. Joffrin \\ CEA Cadarache, 13108 Saint-Paul-lez-Durance, France \\ H. R. Koslowski \\ Forschungszentrum Jülich GmbH, 52425 Jülich, Germany \\ C. Nieswand \\ CRPP/EPFL, 1015 Lausanne, Switzerland \\ S. E. Segre \\ INFM, II Universitá di Roma, Rome and ENEA, CRE Frascati, 00044 Frascati, Italy \\ P. E. Stott \\ JET Joint Undertaking, Abingdon, Oxon, OX14 3EA, United Kingdom \\ C. Walker \\ ITER Joint Central Team, Garching, Germany
}

(Presented on 9 June 1998)

\begin{abstract}
The polarimeter system for ITER features 10 viewing chords covering almost the full poloidal cross section of the plasma in a fan-like arrangement. The 10 laser beams all enter the plasma via $150 \mathrm{~mm}$ diam penetrations through the diagnostic plug at the low-field side. Small retroreflectors at the high-field side mirror the laser beams back towards the penetrations at the low-field side. Extensive feasibility calculations have led to the conclusion that the optimum wavelength for the polarimeter is around $100 \mu \mathrm{m}$. At this wavelength counterproductive effects like refraction and electron cyclotron absorption are negligibly small. However, the anticipated Faraday rotation angles are still very large, such that several important plasma parameters can be measured within the requirements set by the ITER team. In this article the status of the conceptual design of the polarimeter system for ITER will be presented. (C) 1999 American Institute of Physics. [S0034-6748(99)63801-1]
\end{abstract}

\section{INTRODUCTION}

The International Thermonuclear Experimental Reactor (ITER) team has earmarked the current density profile as a potential machine control parameter to be incorporated in feedback loops. Polarimetry has been put forward as a potential technique to measure the current density profile in ITER. An extensive feasibility study by the Microwave Diagnostics group of the European Home Team has led to the conclusion that a poloidal polarimeter system on ITER is, physically speaking, very feasible. ${ }^{1,2}$ Whether such a polarimeter can be successfully operated from the technical point of view is another issue and is addressed in this article.

Results of the feasibility study have already been published elsewhere. ${ }^{1,2}$ In Sec. II we will therefore only summarize the most important results. The technical setup of the polarimeter will be presented in Sec. III, with special emphasis on the geometrical layout, port penetrations through the diagnostic plugs, and retroreflectors at the high-field side. The design work on the polarimeter is still in full progress and therefore the contents of this article should be regarded as a status report. Some of the items that need further R\&D work will be briefly discussed in Sec. IV.

\section{PHYSICAL FEASIBILITY}

Calculations were performed for a range of ITER equilibria and a number of artificially generated density profiles, with shapes ranging from peaked to flat. ${ }^{1,2}$ The line-averaged density was normalized to be a factor of 2 above the Greenwald density for a plasma current of $21 \mathrm{MA}$. Various viewing geometries have been considered ranging from a set of horizontal probing chords to fans of chords entering the blanket through the top port or through the diagnostic plugs in the equatorial port. Three different probing wavelengths have been considered: 10, 50, and $100 \mu \mathrm{m}$.

The main conclusion of the feasibility calculations is that the optimum wavelength for a poloidal polarimeter system is in the order $100 \mu \mathrm{m}$ (e.g., the strong infrared line at $118 \mu \mathrm{m}$ could be used). The Faraday rotation angles can be very large (up to $80^{\circ}$ for single passage through the plasma) and can be easily determined with high accuracy. Even when the polarimeter has a modest accuracy of $2.5 \%$, changes of \pm 0.05 in the central safety factor can be measured. The Cotton-Mouton effect is relatively large and should be corrected for in the analysis of the polarimetry data. However, this should not be regarded as a disadvantage, since it be- 


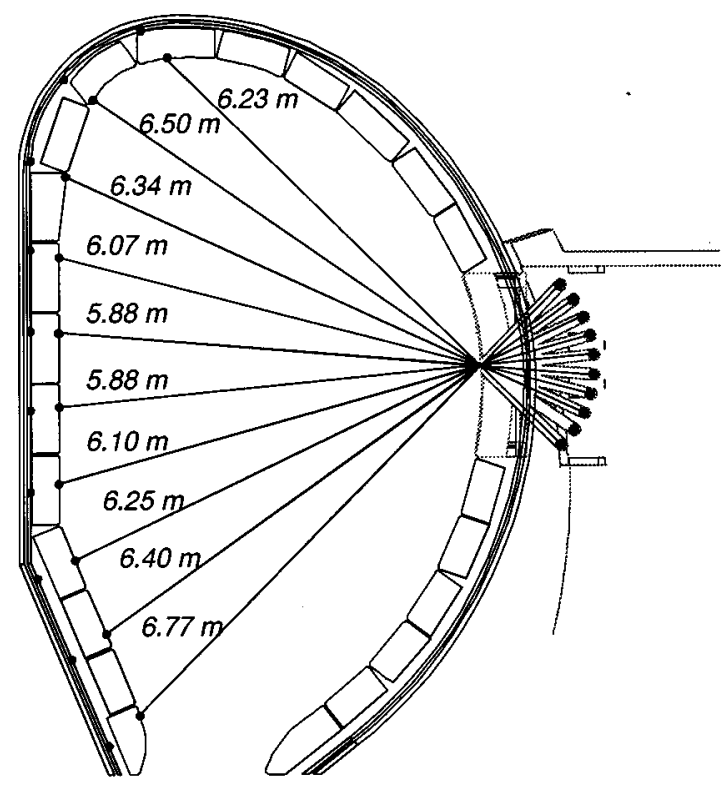

FIG. 1. Geometrical layout of the poloidal polarimeter system for ITER. The distances between the RRs and the port penetration at the LFS are indicated.

comes in principle possible to retrieve the line-averaged density by means of ellipsometry. A proof of principle of this technique has recently been given on the Wendelstein VII-AS stellarator. ${ }^{3}$

Even under the most severe conditions (density a factor of 2 above Greenwald, peaked density profile, and vertical viewing chord), the effect of refraction is negligibly small. The maximum displacement of the chord turned out to be only $6 \mathrm{~mm}$ over a $10 \mathrm{~m}$ long path. Ray tracing for plasmas exhibiting multifaceted asymmetric radiation from the edge (MARFEs) led to the same conclusion. Absorption by very high harmonics of the electron cyclotron frequency is completely negligible under all conditions.

At 10 and $50 \mu \mathrm{m}$ refraction and electron cyclotron absorption are also negligible. However, the Faraday rotation angles at these wavelengths are much smaller (typically $1^{\circ}$ and $20^{\circ}$, respectively, for single passage) and accurate measurements become more difficult. Moreover, one loses the possibility of retrieving the electron density from the Cotton-Mouton effect. For this reason a wavelength near $100 \mu \mathrm{m}$ seems to be optimal. The above results were more or less the same for the various viewing geometries. The Faraday rotation angles, Cotton-Mouton effect, as well as the refraction were slightly, but not significantly, larger for geometries in which the plasma was probed with vertical chords.

\section{TECHNICAL SETUP}

\section{A. Geometrical layout}

Because the precise geometry did not have a large effect on the results of the feasibility calculations, it was decided to choose a geometry that is simplest from the technical point of view (see Fig. 1). A total of 10 probing chords enters the plasma through a diagnostic plug at the low-field side (LFS). The 10 chords form a fan, with a mean crossing point at the plasma facing surface of the blanket. The chords do not have a toroidal component and they cover almost the entire poloidal cross section of the plasma. Small retroreflectors (RRs) are placed at the high-field side (HFS) and mirror the beam back along exactly the same chord through the plasma. The first mirror is placed $1.6 \mathrm{~m}$ behind the plasma facing the surface of the blanket. The second mirror is the only focusing element in the beam transfer system. The beam waist is located at the retroreflector. Various options for the retroreflector will be discussed in Sec. III B, and details of the beam transfer system through the diagnostic plug will be presented in Sec. III C.

\section{B. Retroreflectors at the HFS}

A number of solutions for the RRs at the high-field side has been considered. These included a RR strip, imprinted on the front surface of the blanket tiles at the HFS as well as a RR consisting of six individual corner cubes. These options were discarded since they led to uncontrolled reflections of the beam if it did not hit the RR at the proper position and under the optimal angle.

Therefore, all further investigations have been in RRs consisting of a single corner cube. The ideal position of such a RR would be at the plasma facing side of the blanket modules at the HFS. Because of potential erosion/deposition, the reflector should be somewhat recessed with respect to the front surface. It could be positioned in the gap between two neighboring blanket modules (implying that the sides of these modules should be changed slightly with respect to standard blanket modules). An alternative position would be at the front surface of a single blanket module (for instance, using the holes that are drilled in the blanket modules to mount them onto the stainless steel back plate). Since the ITER Joint Central Team is presently reconsidering the design of the blanket modules, we have not yet put much effort into a detailed design of the RR. Back-to-the-envelope calculations have shown that it is possible to focus the beam to a $10 \mathrm{~mm}$ waist at the HFS. This implies that a RR with a diameter that is a factor of 2.2 larger (e.g., $22 \mathrm{~mm}$ ) would be sufficient to reflect $90 \%$ of the incident radiation (provided alignment is ideal and refraction completely negligible). However, it is advantageous to have a somewhat larger waist at the HFS $(1.5-1.75 \mathrm{~cm})$, since this immediately has a positive effect on the required diameter of the port penetrations at the LFS. Moreover, it is preferrable to have the RRs somewhat larger than a factor 2.2 times the beam waist in order to cope with refraction as well as with small misalignments. An optimal RR diameter would be about $50 \mathrm{~mm}$.

\section{Port penetrations at the LFS}

In Fig. 2 the beam diameter is shown as a function of the distance to the RR. One case is for a beam waist of $10 \mathrm{~mm}$, the other for $17.5 \mathrm{~mm}$. The first mirror in the diagnostic plug is located at a distance of $7.5-8.8 \mathrm{~m}$ from the retroreflector, depending on the chord. The second, focusing mirror is 0.55 $\mathrm{m}$ behind the first mirror, indicated by the vertical dotted 


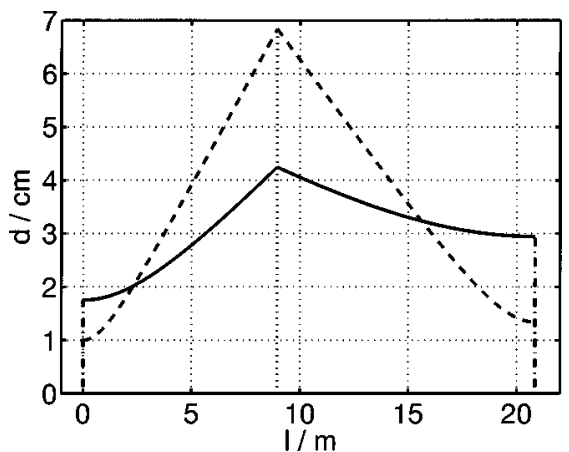

FIG. 2. Calculated values for the beam diameter as a function of the distance to the RR for a beam waist of 10 (dashed line) and $17.5 \mathrm{~mm}$ (solid line), respectively. The vertical dotted line indicates the position of the focusing mirror, the dash-dotted lines indicate the position of the RR and the boxes with beam splitters/combiners, respectively.

line, where the beam diameter is at a maximum. One can see for the $10 \mathrm{~mm}$ beam waist that the beam diameter at the focusing mirror is approximately $70 \mathrm{~mm}$, which means that $180 \mathrm{~mm}$ diam port penetrations would be needed. With a beam waist of $17.5 \mathrm{~mm}$ at the RR, port penetrations with 90-100 mm diameters would in principle be sufficient. However, to enable a certain degree of freedom in the alignment of the beams, the diameter of the penetrations has been fixed at $150 \mathrm{~mm}$. Because the 10 viewing chords cross at the front surface of the blanket at the LFS, the actual port penetration will be an elongated hole $150 \mathrm{~mm}$ wide and $220 \mathrm{~mm}$ high.

To allow sufficient space for other diagnostics, five of the beams are deflected by the first mirror in the current direction, whereas the others are deflected in the countercurrent direction (see Fig. 3). The second mirrors bring the beams back into two planes (each with five beams) that are parallel to the probing plane. In Fig. 3 only the five beams in one of the two planes are shown. The first and second mirrors are mounted in a special mirror assembly that can be easily removed from the diagnostic plug (in Fig. 3 the first and second mirrors are positioned on a line perpendicular to

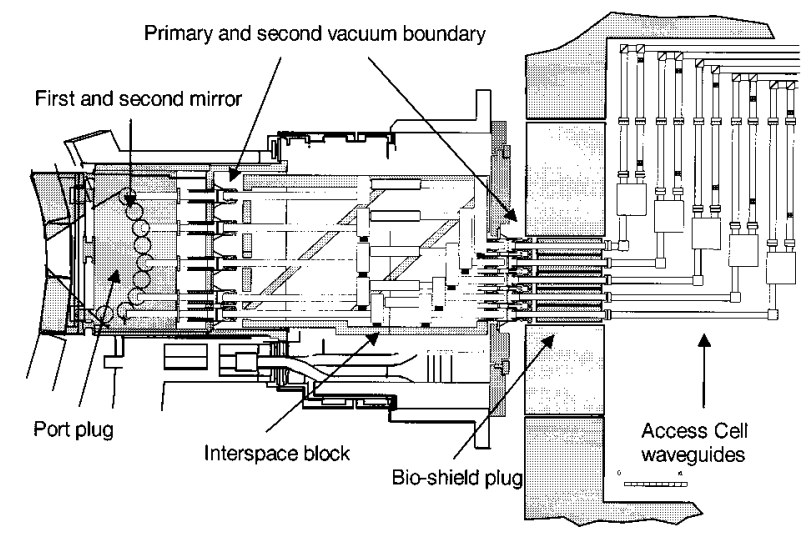

FIG. 3. Schematic drawing of the fully installed equatorial port of the polarimeter. The first and second mirrors of each chord are installed in the port plug, and the interspace block contains the pre-aligned optics to compensate for any relative movements between the port plug and the bioshield. The beam splitters/combiners in the access cell are indicated by the square boxes. From here the waveguides run along the ceiling towards the local diagnostics area where laser(s) and detectors are placed. the plane of the drawing). Special mechanical connections with pushing rods are used to steer the second, focusing mirror for alignment purposes. Quartz vacuum windows are positioned approximately $2-3 \mathrm{~m}$ behind the second mirror to form the primary vacuum boundary. The windows are slightly tilted to avoid any reflections of the incoming beam back into the laser. Quartz has been chosen as the window material because of its low birefringence.

Movements of the diagnostic plug with respect to the cryostat are accommodated by an interspace plug with four mirrors for each chord (see Fig. 3). Once aligned in a testbed, this system maintains its alignment irrespective of any movement at either of its ends. The secondary vacuum boundary, a slightly tilted quartz window, is located directly behind the interspace plug. Straight waveguide sections bring the chords to the access cell outside the bioshield where all the chords are deflected in the vertical direction towards the ceiling of the access gallery. From there, waveguides run along a straight line towards a local diagnostic area where the farinfrared (FIR) laser(s) ${ }^{4}$ and the detectors are placed. Boxes with special optics are positioned in the access cell to separate the two polarization components of the beam as well as to combine the laser beams with the detection lines. The diameter of the waveguides all the way from the port penetration to the access cell is $150 \mathrm{~mm}$. Hence, the waveguides are very oversized, and the actual beam dimensions are determined purely by the focusing optics (see Fig. 2). The second waist of the chords is located in the boxes with beam splitters/combiners. This is done by either adapting the length of the beam paths in the interspace plug or by choosing proper focal lengths of the focusing mirrors for each chord.

\section{Alignment and optical stability}

As was mentioned before, the system features only one focusing element per viewing line. This is the second mirror, which is also the only element that is remotely steerable by pushing rods. The four mirrors (per chord) in the interspace block (see Fig. 3) are aligned in a special testbed. Once aligned, this system maintains its alignment. Mirrors in the access cell, as well as in the local diagnostic area, are steerable remotely. The first and second mirrors that are mounted in a special mirror assembly are pre-aligned in a testbed. However, once they are positioned in the diagnostic plug, they become inaccessible. From that point onward only the second mirror can be used for aligning the beam onto the RRs.

The main alignment problem is to position the beam waist exactly at the center of the RR. To cope with this, the second mirror will be equipped with a scanning mechanism, enabling one to scan the beam over an area with a diameter of approximately $200 \mathrm{~mm}$ at the HFS (slightly different diameters for the various chords). The position where the reflected signal is maximum corresponds to the position of the retroreflector. To enable scanning of the beam over a limited surface at the HFS, the port penetrations through the blanket cannot be smaller than $150 \mathrm{~mm}$.

Vibrations in the mirrors should be at such small levels 
that the beam is not deflected towards a position outside the retroreflector. For the first mirror this means that any angular vibrations should not exceed an angle of $0.1^{\circ}$. To a certain extent vibrations in the first and second mirrors can be compensated for by rigidly attaching both of them to a joint mechanical structure.

\section{E. Detection scheme}

It is too early to make a definite choice for the detection scheme. Various polarimetry techniques have been reported in literature. 5,6 The "classic", ones have a fixed linear input polarization, while the two polarization components in the output beam are measured by individual detectors. More recently techniques have been reported in which the polarization vector of a linearly polarized beam is continuously rotating or in which it is rocking. All of the presently available techniques are compatible with the waveguide system presented above. Therefore, any choice in favor of a certain polarization scheme can be made at a later time.

\section{FUTURE TASKS}

Although the conceptual technical design of the polarimeter seems to be rather well developed, there are still a number of important issues that should be addressed in the near future. Among them are the following:

(1) An assessment of the alignment issues is needed, including extensive simulations with a Gaussian beam raytracing code as well as actual size mock up experiments in a laboratory. Special emphasis should be given to the retroreflector and the alignment and optical stability, especially of the first and second mirrors.

(2) An experimental program should be started to study the effects of erosion and deposition on the optical quality of the RR, as well as to find out whether the RR changes the polarization and ellipticity of the probing beams.

(3) Now the chord geometry of the polarimeter system is more or less fixed; a full assessment of the accuracy and sensitivity of the polarimeter system is needed. This implies that simulations should be done for a variety of ITER equilibria. Special emphasis should be given to find the degree of accuracy in the determination of the central safety factor, the vertical plasma position, and the position (and value) of the minimum $q$ value in case of advanced tokamak operation.

(4) New reconstruction codes should be developed and tested to simultaneously retrieve the current density and electron density profiles from the measured values for the Faraday rotation and ellipticity.

(5) Calculation of the neutron fluence through the penetrations must be done, and the radiation effects on especially the first mirror must be studied.

\section{ACKNOWLEDGMENTS}

Although the authors of this article form the "hard core'" of the European polarimetry team, fruitful use was made of many discussions with a number of colleagues working in the various European institutes. The authors would like to express their gratitude to these colleagues. Special thanks go to J. H. Rommers for presenting this work at the Princeton meeting. The suggestions and sometimes critical remarks by scientists during the various ITER Progress and Expert meetings were also considered to be of great value. The FOM-Instituut voor Plasmafysica Rijnhuizen and Forschungszentrum Jülich $\mathrm{GmbH}$ are partners in the Trilateral Euregio Cluster.

\footnotetext{
${ }^{1}$ C. Nieswand, in Diagnostics for Experimental Thermonuclear Fusion Reactors 2, edited by P. E. Stott, G. Gorini, P. Prandoni, and E. Sindoni (Plenum, New York), p. 213.

${ }^{2}$ A. J. H. Donné, T. Edlington, E. Joffrin, H. R. Koslowski, C. Nieswand, S. Segre, P. Stott, C. Warrick, and V. Zanza, in Ref. 1, p. 203.

${ }^{3}$ Ch. Fuchs and H. Hartfuss, Rev. Sci. Instrum. (these proceedings).

${ }^{4}$ T. Lehecka, Thesis, University of California, Los Angeles, 1986.

${ }^{5}$ A. J. H. Donné, Rev. Sci. Instrum. 66, 3407 (1995).

${ }^{6}$ J. H. Rommers, S. Barry, R. Behn, and Ch. Nieswand, Plasma Phys. Controlled Fusion (submitted).
} 